hep-th/0309039

TIFR/TH/03-16

\title{
Comments on D-brane Interactions in PP-wave Backgrounds
}

\author{
ATISH DABHOLKAR AND JORIS RAEYMAEKERS \\ Department of Theoretical Physics \\ Tata Institute of Fundamental Research \\ Homi Bhabha Road, Mumbai, India 400005. \\ Email: atish, joris@theory.tifr.res.in
}

\begin{abstract}
We calculate the interaction potential between widely separated D-branes in $\mathrm{PP}$-wave backgrounds in string theory as well as in low-energy supergravity. Timelike and spacelike orientations are qualitatively different but in both cases the effective brane tensions and RR charges take the same values as in Minkowski space in accordance with the expectations from the sigma model perturbation theory.
\end{abstract}




\section{Introduction}

In this note, we study the charges and tensions of Dirichlet branes and orientifold planes in plane wave backgrounds $[7,8,9,10]$. In a general curved spacetime, the effective brane tension that is measured from the interaction energy of two widely separated branes is expected to receive $\alpha^{\prime}$ corrections. From the point of view of sigma model perturbation theory, these corrections will be governed by the $\alpha^{\prime}$ corrections to the low energy DBI action and will be given in terms of invariants constructed from the background curvature, field strengths, and the geometric data of the D-brane embedding [4]. Typically, one would also expect corrections that are nonperturbative in $\alpha^{\prime}$. From the point of view of the boundary conformal field theory, the tension of a D-brane is related to the regularized dimension of the state space of the CFT [5]. When formulating string theory in a curved background, it is an important consistency check whether the Dirac quantization condition[2] for the RR charges is satisfied (see [6] for a discussion in the case of branes in group manifolds). The pp-wave background provides another simple example of a background with a nontrivial metric and Ramond-Ramond fields where exact worldsheet computation of the D-brane interaction energy is possible.

We find that the brane tensions for half-BPS D-branes in pp-wave backgrounds are identical to their values in Minkowski space. This nonrenormalization is in accordance with the expectation based on the symmetry and the geometry of the plane wave background but the reasons are different for 'time-like' branes that are longitudinal to the light-cone directions $x^{+}, x^{-}$and for 'space-like' branes that are transverse to the light-cone directions.

Time-like branes have translation invariance along $x^{-}$which implies that the D-brane interacts only with those closed string states that have vanishing $p_{-}$. For these modes, the metric reduces to the Minkowski metric and the background appears flat. Note that this holds for the full interaction potential and not only for widely separated branes. In other words, the one point functions of all closed string modes and not just the massless ones are the same as in Minkowski space. The same result holds for orientifold planes and their interactions with D-branes. It follows that for general orientifolds of the pp-wave background, the orientifold gauge group is the same as in flat space (a particular case was worked out in [24]).

Space-like branes are not translationally invariant along the light-cone directions. In this case, the nonrenormalization follows instead from the special properties of the pp-wave geometry and the fact that the half-BPS branes that we consider here are totally geodesic [11]. In the pp-wave background, all local coordinate invariants constructed out of the background fields vanish. This is essentially because the only nonvanishing components of the background fields have a lower + index and there is no $g^{++}$to contract them. Furthermore, for embeddings that are 
totally geodesic, the second fundamental form vanishes. Using the Gauss-Codazzi equations one can then conclude that all local invariants constructed using the background fields and the embedding geometry also vanish. Hence all $\alpha^{\prime}$ corrections to charge and tension are expected to vanish in this background. This can be checked explicitly to leading order in $\alpha^{\prime}$ using the corrections to the DBI action worked out in $[3,4]$ and is expected to be true to all orders. Note that this argument depends on supersymmetry somewhat indirectly and only to the extent that the embedding of the worldvolume of these branes is required to be totally geodesic in order to preserve half the supersymmetries. Even if the corrections vanish to all order in $\alpha^{\prime}$, there remains the possibility of corrections that are nonperturbative in $\alpha^{\prime}$, but the plane wave geometry is topologically trivial and we do not expect any instanton corrections. It is neverthless important to verify this expectation by an explicit worldsheet computation because the pp-wave background is not a small deformation of Minkowski space in any sense. It is not asymptotically flat and one cannot smoothly interpolate between flat space and the pp-wave by varying a parameter. The dimensionful parameter $\mu$ that is often introduced can be absorbed in a coordinate redefinition and is not a physical parameter of the background. An exact worldsheet computation is therefore desirable to compare the brane tensions in these completely different backgrounds.

We compute the interaction potentials between a pair of branes and a brane and an anti-brane in the pp-wave limit of $A d S_{5} \times S_{5}$ (henceforth denoted by $P P_{10}$ ) and $A d S_{3} \times S_{3} \times R^{4}$ (henceforth denoted by $P P_{6} \times R^{4}$ ). Strings moving in these backgrounds can be quantized in the light-cone Green-Schwarz formalism $[12,13]$. Dbranes in these backgrounds have been constructed in $[14,16,15,17,18,19,11,20]$ and aspects of their interactions were discussed in $[21,22,23]$. The branes we consider here all preserve half of the kinematical and half of the dynamical supersymmetries and can be either spacelike or timelike. We calculate the contribution from the exchange of masssless supergravity modes from the low energy supergravity and DBI action and find that it agrees with the string result to all orders in the parameter $\mu$ provided that the charges and tensions take the same values as in Minkowski space ${ }^{1}$. In agreement with [21], we find that the force between two parallel spacelike D-branes in $P P_{10}$ does not vanish. For spacelike branes in $P P_{6} \times R^{4}$ however, the brane-brane potential is zero. This can be understood from the fermionic zero modes in the open string description.

The computation of interaction energy is of interest also from the point of view of the dual gauge theory. In the dual description, a single D-brane corresponds to a defect conformal field theory (dCFT) [26, 27, 28, 29, 30, 31, 32]. The interaction energy between two D-branes is expected to correspond to the Casimir energy be-

\footnotetext{
${ }^{1}$ In [22], a calculation to leading order in $\mu$ was performed for the D-instanton in $P P_{10}$.
} 
tween the two defects. The precise value of the interaction energy from the string computation thus gives a prediction for the corresponding quantity in the dual theory. Factorizing the string cylinder diagram in the closed string channel gives one point functions of off-shell closed string states emitted from the D-brane. These correspond to one-point functions of various ambient operators in the dCFT. It would be interesting to compare some of these predictions by a gauge theory computation. For timelike branes, the vanishing of the one point functions for closed string states with nonzero $p_{-}$corresponds to the vanishing of one point functions of ambient gauge theory operators with nonzero $J$ charge as a consequence of conservation of $J$ charge in the dCFT. To compare with the nonzero tadpoles of offshell gravitons with vanishing $p_{-}$however would require a nontrivial computation in the $\mathrm{dCFT}$ and we leave this problem for future work.

This note is organized as follows. Space-like branes are discussed in sections $\S 2$ and $\S 3$ and time-like branes are discussed in $\S 4$. The details of the supergravity calculation of the massless exchanges are given in appendix A. The supergravity calculation requires the knowledge of the exact propagators for the tensor mode fluctuations in this background which we derive explicitly in the light-cone gauge.

\section{Spacelike branes in $P P_{10}$}

The $P P_{10}$ background is given by (see appendix A.1 for more details on our conventions):

$$
\begin{aligned}
d s^{2} & =2 d x^{+} d x^{-}-\mu^{2} x^{I} x^{I}\left(d x^{+}\right)^{2}+d x^{I} d x^{I} \\
R_{I++J} & =-\mu^{2} \delta_{I J} \quad R_{++}=8 \mu^{2} \\
F_{+1234} & =F_{+5678}=4 \mu
\end{aligned}
$$

where $I=1 \ldots 8$. The Ramond-Ramond background breaks the $S O(8)$ acting on the $x^{I}$ to $S O(4) \times S O^{\prime}(4)$, the first factor acting on $x^{i}, i=1 \ldots 4$ and the second one acting on $x^{i^{\prime}}, i^{\prime}=5 \ldots 8$. Denoting a spacelike D-brane with $m$ worldvolume directions along the $x^{i}$ and $n$ worldvolume directions along the $x^{i^{\prime}}$ by $(m, n)$, the branes preserving half of the kinematical and half of the dynamical supersymmetries are of the type $(m, m+2)$ (or, equivalently, $(m+2, m))$ with $m=0,1$ or 2 $[14,11]$. Hence we are to consider D1, D3 and D5-branes. These are to be placed at the origin of the $S O(4) \times S O^{\prime}(4)$ directions in order to preserve the aformentioned supersymmetries. We will calculate the interaction energy between pairs of (anti-) D-branes separated along the $x^{+}, x^{-}$directions. 


\subsection{String calculation}

The string theory calculation of the interaction energy between a pair of D-branes of the same dimension was performed in [21]; we will briefly review it here in order to extract the contribution from the lowest lying string modes.

We would like to perform the string calculation in the open string loop channel to get a correctly normalized amplitude. However, in the standard light-cone gauge $X^{+}=p_{-} \tau, X^{ \pm}$are automatically Neumann directions. We can remedy this by using a nonstandard light-cone gauge for the open string $[21,22]$ in which $X^{ \pm}$ are Dirichlet directions. Here, one quantizes the open strings stretching from one brane to the other in the gauge

$$
X^{+}=\frac{r^{+}}{\pi} \sigma
$$

where $r^{+}$is the brane separation along the $x^{+}$coordinate and $\sigma$ is the worldsheet coordinate, $\sigma \in[0, \pi]$. The Virasoro constraints then determine $X^{-}$to be a Dirichlet direction as well ${ }^{2}$. In this gauge, the worldsheet action contains eight massive bosons and fermions with mass

$$
\mathrm{m}=\frac{\mu r^{+}}{\pi}
$$

The interaction energy between branes can be written as

$$
\begin{aligned}
E T & =2 \cdot \frac{1}{2} i \operatorname{Tr}(-1)^{F_{s}} \ln \left(L_{0}-i \epsilon\right) \\
& =i \int_{0}^{\infty} \frac{d s}{s} \operatorname{Tr}(-1)^{F_{s}} e^{-i\left(L_{0}-i \epsilon\right) s}
\end{aligned}
$$

where $F_{s}$ is the spacetime fermion number and the trace is taken in the space of open string states stretching between the branes. $L_{0}$ is the generator of worldheet time translations and can be written as $L_{0}=p_{-} H^{l c}$ with $H^{l c}$ the light-cone Hamiltonian. In writing (2.2), we chose to work in Lorentzian signature for spacetime with a suitable $i \epsilon$ prescrition [33, 34].

For a Dp-brane interacting with an anti-Dp-brane, $L_{0}$ receives a contribution from the separation from the strings being stretched along the transverse directions $x^{+}, x^{-}$and contributions from harmonic oscillators with frequencies $\omega_{k}=\operatorname{sign}(k) \sqrt{k^{2}+\mathrm{m}^{2}}$, where $k$ is integer for the bosonic oscillators and halfinteger for the fermionic ones. The resulting interaction energy is

$$
E_{D p-\bar{D} p}=i \int_{0}^{\infty} \frac{d s e^{-\epsilon s}}{s} e^{-2 \pi i s\left(\frac{2 r^{+} r^{-}}{4 \pi^{2} \alpha^{\prime}}\right)}(2 i \sin \pi \mathrm{m} s)^{3-p}\left(\frac{f_{4}^{(\mathrm{m})}(q)}{f_{1}^{(\mathrm{m})}(q)}\right)^{8},
$$

\footnotetext{
${ }^{2}$ Note that this gauge is consistent with the Virasoro constraints only if the worldsheet is Euclidean [22].
} 
where we have defined modified $f$-functions as in [21]:

$$
\begin{aligned}
& f_{1}^{(\mathrm{m})}(q)=q^{-\Delta_{\mathrm{m}}}\left(1-q^{\mathrm{m}}\right)^{\frac{1}{2}} \prod_{n=1}^{\infty}\left(1-q^{\sqrt{\mathrm{m}^{2}+n^{2}}}\right) \\
& f_{2}^{(\mathrm{m})}(q)=q^{-\Delta_{\mathrm{m}}}\left(1+q^{\mathrm{m}}\right)^{\frac{1}{2}} \prod_{n=1}^{\infty}\left(1+q^{\sqrt{\mathrm{m}^{2}+n^{2}}}\right), \\
& f_{3}^{(\mathrm{m})}(q)=q^{-\Delta_{\mathrm{m}}^{\prime}} \prod_{n=1}^{\infty}\left(1+q^{\sqrt{\mathrm{m}^{2}+(n-1 / 2)^{2}}}\right) \\
& f_{4}^{(\mathrm{m})}(q)=q^{-\Delta_{\mathrm{m}}^{\prime}} \prod_{n=1}^{\infty}\left(1-q^{\sqrt{\mathrm{m}^{2}+(n-1 / 2)^{2}}}\right)
\end{aligned}
$$

and $\Delta_{\mathrm{m}}$ and $\Delta_{\mathrm{m}}^{\prime}$ are defined by

$$
\begin{aligned}
& \Delta_{\mathrm{m}}=-\frac{1}{(2 \pi)^{2}} \sum_{p=1}^{\infty} \int_{0}^{\infty} d s e^{-p^{2} s} e^{-\pi^{2} \mathrm{~m}^{2} / s}, \\
& \Delta_{\mathrm{m}}^{\prime}=-\frac{1}{(2 \pi)^{2}} \sum_{p=1}^{\infty}(-1)^{p} \int_{0}^{\infty} d s e^{-p^{2} s} e^{-\pi^{2} \mathrm{~m}^{2} / s} .
\end{aligned}
$$

For two parallel Dp-branes, the harmonic oscillator frequencies are $\omega_{k}$ with $k$ integer for both the bosons and fermions. The fermionic "zero-modes" have frequency $\mathrm{m}$ and give a nonzero contribution to the interaction energy. The result is

$$
E_{D p-D p}=i \int_{0}^{\infty} \frac{d s e^{-\epsilon s}}{s} e^{-2 \pi i s\left(\frac{2 r^{+} r^{-}}{4 \pi^{2} \alpha^{\prime}}\right)}(2 i \sin \pi \mathrm{m} s)^{3-p}
$$

The large distance behaviour of (2.3) and (2.9) comes from the leading behaviour of the integrand for small $s$. This can be extracted using the modular transformations

$$
f_{1}^{(\mathrm{m})}(s)=f_{1}^{(\widehat{\mathrm{m}})}(-1 / s), \quad f_{2}^{(\mathrm{m})}(s)=f_{4}^{(\widehat{\mathrm{m}})}(-1 / s), \quad f_{3}^{(\mathrm{m})}(s)=f_{3}^{(\widehat{\mathrm{m}})}(-1 / s),
$$

where

$$
\widehat{\mathrm{m}}=i \mathrm{~m} s
$$

This gives the leading behaviour

$$
\begin{aligned}
& E_{D p-\bar{D} p}=-\left(4 \pi^{2} \alpha^{\prime}\right)^{3-p}\left(2 \pi r^{-}\right)^{p-3} \mu^{3-p} \cot ^{4} \mu r^{+} \Gamma(3-p)+\ldots \\
& E_{D p-D p}=-\left(4 \pi^{2} \alpha^{\prime}\right)^{3-p}\left(2 \pi r^{-}\right)^{p-3} \mu^{3-p} \Gamma(3-p)+\ldots
\end{aligned}
$$

The expression in the first line diverges in the flat space limit $\mu \rightarrow 0$; this is the standard divergence due to the infinite volume of the brane. We can separate out 
the volume factor by rewriting the result in terms of the integrated propagator

$I_{0}^{9-p}\left(r^{+}, r^{-}\right)$over the worldvolume directions with the remaining transverse ppwave coordinates set to zero (see in (A.12)):

$$
\begin{aligned}
& E_{D p-\bar{D} p}=-4 \pi\left(4 \pi^{2} \alpha^{\prime}\right)^{3-p} \cos ^{4} \mu r^{+} I_{0}^{9-p}\left(r^{+}, r^{-}\right)+\ldots \\
& E_{D p-D p}=-4 \pi\left(4 \pi^{2} \alpha^{\prime}\right)^{3-p} \sin ^{4} \mu r^{+} I_{0}^{9-p}\left(r^{+}, r^{-}\right)+\ldots
\end{aligned}
$$

One recovers the correct flat space expression [1] as $\mu \rightarrow 0$ taking into account that, from (A.8),

$$
\lim _{\mu \rightarrow 0} I_{0}^{9-p}\left(r^{+}, r^{-}\right)=V_{p+1} G_{0}^{9-p}\left(r^{+}, r^{-}\right)
$$

where $V_{p+1}$ is the divergent D-brane volume and $G_{0}^{9-p}\left(r^{+}, r^{-}\right)$stands for the Minkowski space scalar propagator integrated over the worldvolume directions.

\section{$2.2 \quad$ Field theory calculation}

We will presently see how the long-range potentials (2.13) are reproduced exactly from the type IIB supergravity action (A.1) supplemented with D-brane source terms

$$
S_{p}=-T_{p} \int d^{p+1} x \sqrt{-\tilde{g}} e^{\frac{p-3}{4} \Phi}+\mu_{p} \int A_{[p+1]}
$$

where $\tilde{g}$ stands for the induced metric on the worldvolume and $T_{p}$ and $\mu_{p}$ are the brane tension and $\mathrm{RR}$ charge respectively. In appendix A.1 we expand the bulk action to quadratic order in the fluctuations around the $P P_{10}$ background, adopting the light-cone gauge for the fluctuations. The resulting action is a sum of decoupled terms characterized by an integer $c$ :

$$
S_{\psi}=\frac{1}{4 \kappa^{2}} \int d^{10} x \psi^{\dagger}\left(\square-2 i \mu c \partial_{-}\right) \psi
$$

In general, $\psi$ is in a tensor representation of $S O(4) \times S O^{\prime}(4)$ and a contraction of tensor indices is understood. The decoupled fields $\psi$, their values of $c$ and their $S O(4) \times S O^{\prime}(4)$ representations are given in the appendix in table 1. Expanding the source action (2.14) to linear order in the fluctuations we get source terms for the components $\psi_{\alpha}$ of the form

$$
S_{\text {source }}=\int d^{10} x \delta^{9-p}\left(x-x_{0}\right) k\left(\psi_{\alpha}+\epsilon \bar{\psi}_{\alpha}\right)
$$

where $k$ is a constant proportional to either $T_{p}$ or $\mu_{p}$ and $\epsilon= \pm 1$. The contribution of such a mode to the interaction energy can then be written in terms of the 
integrated propagator and the constants $(c, k, \epsilon)$. For example, if $\psi$ is an $S O(4) \times$ $S O^{\prime}(4)$ singlet one gets a contribution to the interaction energy

$$
E_{(c, k, \epsilon)}=8 \epsilon \kappa^{2} k^{2} \cos \mu c r^{+} I_{0}^{9-p}\left(r^{+}, r^{-}\right) .
$$

When $\psi$ is in a tensor representation $S O(4) \times S O^{\prime}(4)$, this expression can get an extra overall factor from the fact that one has to use a propagator with the right symmetry properties.

\subsubsection{D1-brane}

We can take the worldvolume along the directions $x^{1}, x^{2}$. The source terms are given by

$$
\begin{aligned}
\mathcal{L}_{\text {source }} & =\frac{i T_{1}}{2}\left(h_{11}+h_{22}-\phi\right) \pm \mu_{1} a_{12} \\
& =\frac{i T_{1}}{\sqrt{2}}\left(h_{11}^{\perp}+h_{22}^{\perp}\right)+\frac{i T_{1}}{4}(H+\bar{H})-\frac{i T_{1}}{2} \phi+\frac{i T_{1}}{4} h \pm \frac{\mu_{1}}{\sqrt{2}}\left(G_{12}+\bar{G}_{12}\right)
\end{aligned}
$$

where the upper (lower) sign applies to a brane (antibrane) source. The factors of $i$ multiplying the tension arise because $-\tilde{g}$ is negative for spacelike branes. The trace $h$ doesn't propagate in the light-cone gauge. The constants $(c, k, \epsilon)$ for the other source terms are summarized in the following table:

\begin{tabular}{|c||c|c|c|c|}
\hline$\psi$ & $h_{11}^{\perp}, h_{22}^{\perp}$ & $H$ & $\phi$ & $G_{12}$ \\
\hline$(c, k, \epsilon)$ & $\left(0, \frac{i T_{1}}{2 \sqrt{2}}, 1\right)$ & $\left(4, \frac{i T_{1}}{4}, 1\right)$ & $\left(0, \frac{-i T_{1}}{4}, 1\right)$ & $\left(2, \frac{ \pm \mu_{1}}{\sqrt{2}}, 1\right)$ \\
\hline
\end{tabular}

Summing up all contributions to the interaction energy gives

$$
E=-4 \kappa^{2}\left[\frac{T_{1}^{2}}{8} \cos 4 \mu r^{+}+\frac{3}{8} T_{1}^{2} \mp \frac{\mu_{1}^{2}}{2} \cos 2 \mu r^{+}\right] I_{0}^{8}\left(r^{+}, r^{-}\right)
$$

where the upper (lower) sign applies to the brane-brane (brane-antibrane) system.

\subsubsection{D3-brane}

We take the worldvolume directions to be $x^{1}, x^{2}, x^{3}, x^{5}$. The source terms are

$$
\begin{aligned}
\mathcal{L}_{\text {source }} & =\frac{i T_{3}}{2}\left(h_{11}+h_{22}+h_{33}+h_{55}\right)+\mu_{3} a_{1235} \\
& =\frac{i T_{3}}{\sqrt{2}}\left(h_{11}^{\perp}+h_{22}^{\perp}+h_{33}^{\perp}+h_{55}^{\perp}\right)+\frac{i T_{3}}{4}(H+\bar{H})+\frac{1}{2} h \mp \frac{i \mu_{3}}{2}\left(H_{45}-\bar{H}_{45}\right)
\end{aligned}
$$

These give the following interaction energy contributions 


\begin{tabular}{|c||c|c|c|}
\hline$\psi$ & $h_{11}^{\perp}, h_{22}^{\perp}, h_{33}^{\perp}, h_{55}^{\perp}$ & $H$ & $H_{45}$ \\
\hline$(c, k, \epsilon)$ & $\left(0, \frac{i T_{3}}{2 \sqrt{2}}, 1\right)$ & $\left(4, \frac{i T_{3}}{4}, 1\right)$ & $\left(2, \frac{\mp i \mu_{3}}{2},-1\right)$ \\
\hline
\end{tabular}

The total is

$$
E=-4 \kappa^{2}\left[\frac{T_{3}^{2}}{8} \cos 4 \mu r^{+}+\frac{3 T_{3}^{2}}{8} \mp \frac{\mu_{3}^{2}}{2} \cos 2 \mu r^{+}\right] I_{0}^{6}\left(r^{+}, r^{-}\right)
$$

\subsubsection{D5-brane}

The calculation is the same as for the D1-brane due to S-duality invariance of the type IIB supergravity action and the fact that the $P P_{10}$ background is also S-duality invariant. The end result is again

$$
E=-4 \kappa^{2}\left[\frac{T_{5}^{2}}{8} \cos 4 \mu r^{+}+\frac{3 T_{5}^{2}}{8} \mp \frac{\mu_{5}^{2}}{2} \cos 2 \mu r^{+}\right] I_{0}^{4}\left(r^{+}, r^{-}\right)
$$

\subsubsection{D-brane charges and tensions}

Comparing the string calculation (2.13) with the field theory results $(2.18,2.19$, 2.20 ) we find agreement only if the charges and tensions are equal and their numerical value is the same as in Minkowski space [1]:

$$
T_{p}^{2}=\mu_{p}^{2}=\frac{\pi\left(4 \pi^{2} \alpha^{\prime}\right)^{(3-p)}}{\kappa^{2}} \quad p=1,3,5 .
$$

To see this, one has to use the trigonometric identities

$$
\begin{aligned}
\cos ^{4} x & =\frac{1}{8} \cos 4 x+\frac{3}{8}+\frac{1}{2} \cos 2 x \\
\sin ^{4} x & =\frac{1}{8} \cos 4 x+\frac{3}{8}-\frac{1}{2} \cos 2 x .
\end{aligned}
$$

These identities in a sense encode the equivalence between the open- and closed string descriptions and were instrumental in proving Cardy's condition for boundary states in [21].

\section{Spacelike branes in $P P_{6} \times R^{4}$}

Our conventions for the $P P_{6} \times R^{4}$ coordinates and background fields are (see appendix A.3 for more details)

$$
\begin{aligned}
d s^{2} & =2 d x^{+} d x^{-}-\mu^{2}(z \bar{z}+w \bar{w})\left(d x^{+}\right)^{2}+d z d \bar{z}+d w d \bar{w}+d x^{a} d x^{a} \\
R_{z++\bar{z}} & =-\frac{1}{2} \mu^{2} \quad R_{w++\bar{w}}=-\frac{1}{2} \mu^{2} \quad R_{++}=4 \mu^{2} \\
F_{+z \bar{z}} & =F_{+w \bar{w}}=i \mu
\end{aligned}
$$


The allowed D-branes in $P P_{6} \times R^{4}$ were classified in [16]. Here, we restrict attention to spacelike branes with worldvolumes lying in the $P P_{6}$ part of the geometry. Branes with worldvolume directions along the $R^{4}$ (and their tensions) can be obtained by applying T-duality along the $R^{4}$ directions. Denoting by $(m, n)$ a brane with $m$ directions along the $U(1)$ and $n$ directions along $U^{\prime}(1)$, the branes preserving half the kinematical and half the dynamical supersymmetries are of the type $(m, m)$ with $m=1,2$. This leaves the D1 and D3 branes to be considered. Supersymmetry requires that the D1-brane be placed at the origin of the transverse $U(1) \times U^{\prime}(1)$ directions.

\subsection{String calculation}

The string theory calculation of the interaction energy proceeds as in the $P P_{10}$ case. After fixing a non-standard light-cone gauge, the worldsheet action for strings stretching between branes contains four massive bosons and fermions with mass

$$
\mathrm{m}=\frac{\mu r^{+}}{\pi}
$$

as well as four massless bosons and fermions. The interaction energy between a Dp-brane and an anti-Dp-brane is given by the open string one-loop amplitude

$$
E_{D p-\bar{D} p}=i \int_{0}^{\infty} \frac{d s e^{-\epsilon s}}{s} e^{-2 \pi i s\left(\frac{2 r^{+} r^{-}+r^{a} r^{a}}{4 \pi^{2} \alpha^{\prime}}\right)}(2 i \sin \pi \mathrm{m} s)^{1-p}\left(\frac{f_{4}^{(\mathrm{m})}(q)}{f_{1}^{(\mathrm{m})}(q)}\right)^{4}\left(\frac{f_{4}^{(0)}(q)}{f_{1}^{(0)}(q)}\right)^{4}
$$

The leading contribution comes from massless exchanges and is given by

$$
E_{D p-\bar{D} p}=-4 \pi\left(4 \pi^{2} \alpha^{\prime}\right)^{3-p} \cos ^{2} \mu r^{+} I_{0}^{9-p}\left(r^{+}, r^{-}, r^{a}\right)
$$

where $I_{0}^{9-p}\left(r^{+}, r^{-}, r^{a}\right)$ stands for the scalar propagator integrated over $p+1$ longitudinal D-brane directions with the remaining transverse pp-wave coordinates set to zero (see (A.20)). We again observe that (3.3) reduces to the correct flat-space expression [1] as $\mu \rightarrow 0$.

Contrary to the $P P_{10}$ case, the interaction energy between two parallel branes in $P P_{6} \times R^{4}$ is zero:

$$
E_{D p-D p}=0 .
$$

This follows immediately from the fact that an open string stretching between the branes has four actual (meaning zero-frequency) fermionic zero modes. 


\subsection{Field theory calculation}

In appendix A.3 we expand the bosonic type IIB action around the $P P_{6} \times R^{4}$ background and identify the independent fluctuations. The field theory calculation of the interaction energy again reduces to a sum of contributions of the form (2.17), characterized by constants $(c, k, \epsilon)$ which can be read off by writing the D-brane source terms in terms of the decoupled fields listed in table 2 of the appendix.

\subsubsection{D1-brane}

We take the worldvolume directions to be $x^{1}, x^{3}$. Expressing the D-brane sources in terms of the decoupling fields in table 2 one gets the worldvolume Lagrangian

$$
\begin{aligned}
\mathcal{L}_{\text {source }} & =\frac{i T_{1}}{2}\left(h_{11}+h_{33}-\phi\right) \pm \mu_{1} a_{13} \\
& =\frac{i T_{1}}{4}\left(\tilde{h}_{z z}+\tilde{h}_{\bar{z} \bar{z}}+\tilde{h}_{w w}+\tilde{h}_{\bar{w} \bar{w}}+H+\bar{H}+H^{\prime}+\bar{H}^{\prime}\right)+\frac{i T_{1}}{4} h \\
& \pm \frac{\mu_{1}}{4}\left(H_{z w}^{+}+H_{\bar{z} \bar{w}}^{+}-H_{z w}^{-}-H_{\bar{z} \bar{w}}^{-}+H_{z \bar{w}}^{+}+H_{\bar{z} w}^{+}-H_{z \bar{w}}^{-}-H_{\bar{z} w}^{-}\right) .
\end{aligned}
$$

where the upper sign refers to a brane and the lower one to an anti-brane. The trace $h$ does not propagate in the light-cone gauge. The other fields give contributions of the form (2.17) to the interaction energy. These are summarized in the following table:

\begin{tabular}{|c||c|c|c|c|c|}
\hline$\psi$ & $\tilde{h}_{z z}, \tilde{h}_{w w}$ & $H, H^{\prime}$ & $H_{z w}^{+}, H_{z w}^{-}$ & $H_{z \bar{w}}^{+}$ & $H_{z \bar{w}}^{-}$ \\
\hline$(c, k, \epsilon)$ & $\left(0, \frac{i T_{1}}{4}, 1\right)$ & $\left(2, \frac{i T_{1}}{4}, 1\right)$ & $\left(0, \frac{ \pm \mu_{1}}{4}, 1\right)$ & $\left(2, \frac{ \pm \mu_{1}}{4}, 1\right)$ & $\left(-2, \frac{ \pm \mu_{1}}{4}, 1\right)$ \\
\hline
\end{tabular}

where the upper sign applies to the brane-brane system and the lower sign applies to the brane-antibrane configuration. Summing all contributions, one gets the total interaction energy

$$
\begin{aligned}
E & =-2 \kappa^{2}\left[T_{1}^{2}\left(\cos 2 \mu r^{+}+1\right) \mp \mu_{1}^{2}\left(\cos 2 \mu r^{+}+1\right)\right] I_{0}^{8}\left(r^{+}, r^{-}, r^{a}\right) \\
& =-2 \kappa^{2}\left[T_{1}^{2} \mp \mu_{1}^{2}\right] \cos ^{2} \mu r^{+} I_{0}^{8}\left(r^{+}, r^{-}, r^{a}\right)
\end{aligned}
$$

\subsubsection{D3-brane}

The worldvolume directions are $x^{1}, x^{2}, x^{3}, x^{4}$. The source terms are

$$
\begin{aligned}
\mathcal{L}_{\text {source }} & =\frac{i T_{3}}{2}\left(h_{11}+h_{22}+h_{33}+h_{44}\right) \pm \mu_{3} a_{1234} \\
& =\frac{i T_{3}}{4}\left(H+\bar{H}+H^{\prime}+\bar{H}^{\prime}\right)+\frac{i T_{3}}{\sqrt{2}} H_{0}+\frac{i T_{3}}{2} h \\
& \pm \frac{\mu_{3}}{2 \sqrt{2}}(G+\bar{G}) \pm \frac{\mu_{3}}{\sqrt{2}} G_{0} .
\end{aligned}
$$


The contributions to the interaction energy are summarized in the following table:

\begin{tabular}{|c||c|c|c|c|}
\hline$\psi$ & $H, H^{\prime}$ & $H_{0}$ & $G, G^{\prime}$ & $G_{0}$ \\
\hline$(c, k, \epsilon)$ & $\left(2, \frac{i T_{3}}{4}, 1\right)$ & $\left(0, \frac{i T_{3}}{2 \sqrt{2}}, 1\right)$ & $\left(2, \frac{ \pm \mu_{3}}{2 \sqrt{2}}, 1\right)$ & $\left(0, \frac{ \pm \mu_{3}}{2 \sqrt{2}}, 1\right)$ \\
\hline
\end{tabular}

Summing all contributions, one gets the total interaction energy

$$
\begin{aligned}
E & =-2 \kappa^{2}\left[T_{3}^{2}\left(\cos 2 \mu r^{+}+1\right) \mp \mu_{3}^{2}\left(\cos 2 \mu r^{+}+1\right)\right] I_{0}^{6}\left(r^{+}, r^{-}, r^{a}\right) \\
& =-2 \kappa^{2}\left[T_{3}^{2} \mp \mu_{3}^{2}\right] \cos ^{2} \mu r^{+} I_{0}^{6}\left(r^{+}, r^{-}, r^{a}\right)
\end{aligned}
$$

Again, the upper sign applies to the brane-brane system and the lower sign applies to the brane-antibrane configuration.

\subsection{D-brane charges and tensions}

Comparing the results (3.3) and (3.4) of the string calculation with the field theory results (3.6) and (3.8), we find the value of the D-brane charge and tension:

$$
T_{p}^{2}=\mu_{p}^{2}=\frac{\pi\left(4 \pi^{2} \alpha^{\prime}\right)^{(3-p)}}{\kappa^{2}} \quad p=1,3 .
$$

These values are again the same as in Minkowski space.

\section{Timelike Branes}

In this section we will argue that interactions between timelike D-branes that extend along the $x^{+}, x^{-}$directions in a plane wave geometry are the same as in Minkowski geometry. This is a consequence of the fact that these branes preserve translation invariance in the $x^{-}$direction. Similar results hold for orientifold planes.

For definiteness, we illustrate this in the $P P_{6} \times R^{4}$ background and comment on the generalization to other pp-wave backgrounds at the end of this section. Let us consider a brane-brane or a brane-anti-brane pair in this background separated along the $R^{4}$ directions. From the point of view of the low-energy effective field theory, the long-range interaction potential comes from the exchange of massless modes between the branes. The Feynman propagator $G_{c}\left(x_{1}, x_{2}\right)$ of such modes is given by (see (A.17))

$$
\sum_{\mathbf{n}} \int \frac{d p_{+} d p_{-} d^{4} p_{a}}{(2 \pi)^{6}} \frac{e^{i\left(p_{+}\left(x_{1}^{+}-x_{2}^{+}\right)+p_{-}\left(x_{1}^{-}-x_{2}^{-}\right)+p_{a}\left(x_{1}^{a}-x_{2}^{a}\right)\right)} \psi_{\mathbf{n}}^{\left(\mu p_{-}\right)}\left(x_{1}^{A}\right) \psi_{\mathbf{n}}^{\left(\mu p_{-}\right)}\left(x_{2}^{A}\right)}{2 p_{+} p_{-}+\mu p_{-} \sum_{I}\left(n_{I}+\frac{1}{2}\right)+2 c \mu p_{-}+p_{a} p_{a}-i \epsilon} .
$$


In calculating the interaction energy between a pair of branes, we have to integrate (A.8) over the worldvolume directions which include $x_{1}^{-}, x_{2}^{-}$. This gives a delta function for $p_{-}$, hence the result is independent of $\mu$. We recover the flat space result for the interaction energy (times the interaction time):

$$
E T=2 \kappa^{2}\left(T_{p}^{2} \mp \mu_{p}^{2}\right) G_{9-p}\left(r^{a}\right) V_{p+1}
$$

A similar argument can be made for the exchange of massive modes, which shows that the full brane-antibrane interaction potential is the same as in Minkowski space. In the language of boundary states ${ }^{3}$, The interaction energy, say, between a brane and an anti-brane is given by the overlap $\langle D \bar{p}|\Delta| D p\rangle$ where $\Delta$ is the closed string propagator and $|D p\rangle,|D \bar{p}\rangle$ are the boundary states. Since the boundary states satisfy $p_{-}|D p\rangle=p_{-}|D \bar{p}\rangle=0$, the closed string propagator is projected on the $p_{-}=0$ subspace and these states propagate as in flat space.

It is instructive to see how the same conclusion follows from a calculation in the open string picture. This, in some sense, is the natural picture to use for timelike branes since the open strings can be quantized in the usual light-cone gauge $X^{+}=p_{-} \tau$. In this gauge, the coordinates $X^{ \pm}$automatically obey Neumann boundary conditions [14]. The brane-antibrane interaction energy is given by

$$
E T=i V_{+-} \int_{0}^{\infty} \frac{d s e^{-\epsilon s}}{s} \int \frac{d p_{+} d p_{-}}{(2 \pi)^{2}} e^{-4 \pi \alpha^{\prime} i s p_{+} p_{-}} Z\left(s, \mu p_{-}\right)
$$

where

$$
\begin{aligned}
Z\left(s, \mu p_{-}\right) & =\operatorname{Tr}(-1)^{F_{s}} q^{\alpha^{\prime} p_{-} H^{\mathrm{lc}}} \\
& =q^{\frac{r^{a} a^{a}}{(2 \pi)^{2} \alpha^{\prime}}}\left(2 i \sin \pi \mu p_{-} s\right)^{3-p}\left(\frac{f_{4}^{\left(\mu p_{-}\right)}(q) f_{4}^{(0)}(q)}{f_{1}^{\left(\mu p_{-}\right)}(q) f_{1}^{(0)}(q)}\right)^{4} .
\end{aligned}
$$

The function $Z\left(s, \mu p_{-}\right)$is the partition function for a combined system of four massive scalars and fermions with mass $\mu p_{-}$and four massless scalars and fermions, with appropriate boundary conditions. Returning to $(4.2)$, we see that the $p_{+}$ integral yields a delta function. Integrating over $p_{-}$we find

$$
E T=i V_{+-} \int_{0}^{\infty} \frac{d s e^{-\epsilon s}}{8 \pi^{2} \alpha^{\prime} s^{2}} Z(s, 0) .
$$

In particular, using a modular transformation to extract the small $s$ behaviour of the integrand, one finds the dominating contribution for widely separated branes

$$
E T=4 \pi\left(4 \pi^{2} \alpha^{\prime}\right)^{(3-p)} V_{p+1} G_{9-p}\left(r^{a}\right)+\ldots
$$

\footnotetext{
${ }^{3}$ The only subtlety here is that one has to quantize the closed string in a nonstandard lightcone gauge in order for the coordinates $X^{ \pm}$to have the right boundary conditions [21].
} 
Comparing with (4.1) we find

$$
T_{p}^{2}=\mu_{p}^{2}=\frac{\pi\left(4 \pi^{2} \alpha^{\prime}\right)^{(3-p)}}{\kappa^{2}}
$$

which is indeed the flat-space value [1].

Similar results hold for the interactions between timelike orientifold planes, and between orientifold planes and D-branes in $P P_{6} \times R^{4}$. This can be argued from the fact that the crosscap state is annilated by $p_{-}$or, in the open string picture, from the fact that the interaction energy is again of the form (4.2) but with a different function $Z\left(s, \mu p_{-}\right)[24,25]$. Hence the tadpole cancellation conditions for timelike orientifolds in $P P_{6} \times R^{4}$ are the same as in Minkowski space. The above argument shows that not only the massless tadpoles but the one point functions on a disk of even the massive string modes take the same value as in Minkowski space.

\section{Acknowledgements}

We would like to thank Kostas Skenderis and Marika Taylor for very useful discussions and comments.

\section{A Massless modes and propagators in $P P_{6}$ and $P P_{10}$}

In this appendix we obtain the Lagrangian and the propagator for the bosonic massless supergravity modes in the pp-wave background. The starting point is the bosonic part of the type IIB action in the Einstein frame:

$$
\begin{aligned}
S= & \frac{1}{2 \kappa^{2}} \int d^{10} x \sqrt{-g}\left[R-\frac{1}{2}(\partial \Phi)^{2}-\frac{H_{[3]}^{2}}{2 \cdot 3 !}-\frac{1}{2} e^{-2 \Phi} F_{[1]}^{2}-\frac{e^{-\Phi} \tilde{F}_{[3]}^{2}}{2 \cdot 3 !}-\frac{\tilde{F}_{[5]}^{2}}{4 \cdot 5 !}\right] \\
& -\frac{1}{2} \int A_{[4]} \wedge H_{[3]} \wedge F_{[3]}
\end{aligned}
$$

where $F_{[2 n+1]} \equiv d A_{[2 n]}, H_{[3]} \equiv d B_{[2]}$ and

$$
\begin{aligned}
\tilde{F}_{[3]} & \equiv F_{[3]}-A_{[0]} \wedge H_{[3]} \\
\tilde{F}_{[5]} & \equiv F_{[5]}-\frac{1}{2} A_{[2]} \wedge H_{[3]}+\frac{1}{2} B_{[2]} \wedge F_{[3]}
\end{aligned}
$$

In the following we will expand the action around the $P P_{6}$ and $P P_{10}$ backgrounds to quadratic order in the fluctuations

$$
g_{\mu \nu} \rightarrow g_{\mu \nu}+h_{\mu \nu}
$$




$$
\begin{aligned}
\Phi & \rightarrow \Phi+\phi \\
B_{[2]} & \rightarrow B_{[2]}+b_{[2]} \\
A_{[2 n]} & \rightarrow A_{[2 n]}+a_{[2 n]} .
\end{aligned}
$$

It's convenient to split the metric fluctuations into a trace part $h$ and a traceless tensor $h_{\mu \nu}^{T}$. We adopt the light-cone gauge for the fluctuations:

$$
h_{-\mu}=b_{-\mu}=a_{-\mu_{1} \ldots \mu_{2 n-1}}=0 .
$$

In this gauge, after shifting the fields with $\mathrm{a}_{+}$index, one finds that $h, h_{+\mu}^{T}, b_{+\mu}$ and $a_{+\mu_{1} \ldots \mu_{2 n-1}}$ decouple. This situation is familiar from the light-cone gauge in Minkowski space (see e.g. [36]). Hence the only propagating fields are the transverse modes $h_{I J}, b_{I J}, a_{I_{1} \ldots I_{2 n}} ; I, J, \ldots=1 \ldots 8$. In the presence of general sources, the gauge-fixed Lagrangian contains Coulomb-like terms as a result of shifting the

fields. In the cases we consider, these are absent because spacelike branes do not provide a source for the fields with $\mathrm{a}+$ index.

\section{A.1 Massless modes in $P P_{10}$}

We use the following index conventions:

$$
\begin{array}{rl}
\mu, \nu, \ldots=0, \ldots, 9 & \mathrm{SO}(9,1) \text { vector indices } \\
I, J, \ldots=1, \ldots 8 & \mathrm{SO}(8) \text { vector indices } \\
i, j, \ldots=1, \ldots 4 & \mathrm{SO}(4) \text { vector indices } \\
i^{\prime}, j^{\prime}, \ldots=5, \ldots 8 & \mathrm{SO}^{\prime}(4) \text { vector indices }
\end{array}
$$

The nonvanishing $P P_{10}$ background fields are given by

$$
\begin{aligned}
d s^{2} & =2 d x^{+} d x^{-}-\mu^{2} x^{I} x^{I}\left(d x^{+}\right)^{2}+d x^{I} d x^{I} \\
R_{I++J} & =-\mu^{2} \delta_{I J} \quad R_{++}=8 \mu^{2} \\
F_{+1234} & =F_{+5678}=4 \mu
\end{aligned}
$$

In light-cone gauge, the $S O(4) \times S O^{\prime}(4)$ subgroup of the background symmetry group is manifest with $x^{i}$ and $x^{i^{\prime}}$ transforming as vectors under $S O(4)$ and $S O^{\prime}(4)$ respectively.. Expanding the action (A.1) around this background to quadratic order one can organize the fluctuations into (complex) decoupled fields $\psi$ which transform in irreducible representations of $S O(4) \times S O^{\prime}(4)$ [12]. We choose our normalizations so that each field $\psi$ contributes a term to the Lagrangian density of the form

$$
\mathcal{L}=\frac{1}{4 \kappa^{2}} \bar{\psi}\left(\square-2 i \mu c \partial_{-}\right) \psi
$$




\begin{tabular}{|ccc|c|c|}
\hline$\psi$ & & linear combination & c & irrep \\
\hline \hline$h_{i j}^{\perp}$ & $=$ & $\frac{1}{\sqrt{2}}\left(h_{i j}^{T}-\frac{1}{4} \delta_{i j} h_{k k}^{T}\right)$ & 0 & $(\mathbf{9 , 1})$ \\
$h_{i^{\prime} j^{\prime}}^{\perp}$ & $=$ & $\frac{1}{\sqrt{2}}\left(h_{i^{\prime} j^{\prime}}^{T}+\frac{1}{4} \delta_{i^{\prime} j^{\prime}} h_{k k}^{T}\right)$ & 0 & $(\mathbf{1 , 9})$ \\
$H$ & $=$ & $\frac{1}{2}\left(h_{i i}^{T}+\frac{i}{12} a_{i j k l} \epsilon_{i j k l}\right)$ & 4 & $(\mathbf{1}, \mathbf{1})$ \\
$H_{i i^{\prime}}$ & $=$ & $h_{i i^{\prime}}^{T}+\frac{i}{6} a_{i^{\prime} j k l} \epsilon_{i j k l}$ & 2 & $(\mathbf{4 , 4 )}$ \\
$G_{i j}$ & $=$ & $\frac{1}{\sqrt{2}} a_{i j}+\frac{i}{2 \sqrt{2}} \epsilon_{i j k l} b_{k l}$ & 2 & $(\mathbf{6 , 1})$ \\
$G_{i^{\prime} j^{\prime}}$ & $=$ & $\frac{1}{\sqrt{2}} a_{i^{\prime} j^{\prime}}+\frac{i}{2 \sqrt{2}} \epsilon_{i^{\prime} j^{\prime} k^{\prime} l^{\prime}} b_{k^{\prime} l^{\prime}}$ & 2 & $(\mathbf{1 , 6 )}$ \\
& $b_{i j^{\prime}}$ & & 0 & $(\mathbf{4 , 4})$ \\
& & & 0 & $(\mathbf{4 , 4})$ \\
& $a_{i j^{\prime}}$ & & 0 & $(\mathbf{1 , 1})$ \\
\hline$\phi$ & & &
\end{tabular}

Table 1: Decoupled massless fields in $P P_{10}$.

where contractions of the $S O(4) \times S O^{\prime}(4)$ indices are implied where appropriate and the bar denotes complex conjugation. The operator $\square=2 \partial_{+} \partial_{-}+\mu^{2} x^{I} x^{I} \partial_{-}^{2}+\partial_{I} \partial_{I}$ is the scalar Laplacian in $P P_{10}$ and $c$ is an integer. The results needed for the calculation of the D-brane tensions are summarized in table 1. It displays the fields $\psi$, their definition in terms of the original fluctuations (A.3), their value of $c$ and their irrep of $S O(4) \times S O^{\prime}(4)$.

\section{A.2 Massless propagators in $P P_{10}$}

We will also need the Feynman propagator $G_{c}\left(x_{1}, x_{2}\right)$ corresponding to the operator $\square-2 i \mu c \partial_{-}$. It can be written as

$$
G_{c}\left(x_{1}, x_{2}\right)=-i \sum_{\mathbf{n}} \int \frac{d p_{+} d p_{-}}{(2 \pi)^{2}} \frac{e^{i\left(p_{+}\left(x_{1}^{+}-x_{2}^{+}\right)+p_{-}\left(x_{1}^{-}-x_{2}^{-}\right)\right)} \psi_{\mathbf{n}}^{\left(\mu p_{-}\right)}\left(x_{1}^{I}\right) \psi_{\mathbf{n}}^{\left(\mu p_{-}\right)}\left(x_{2}^{I}\right)}{2 p_{+} p_{-}+\mu p_{-} \sum_{I}\left(n_{I}+\frac{1}{2}\right)+2 c \mu p_{-}+p_{I} p_{I}-i \epsilon}
$$

Here, $\mathbf{n}=\left(n_{1}, n_{2}, \ldots, n_{8}\right)$, and $\psi_{\mathbf{n}}^{(\mathrm{m})}$ is a product of normalized harmonic oscillator eigenfunctions satisfying $\left(-\partial_{I} \partial_{I}+\mathrm{m}^{2}\right) \psi_{\mathbf{n}}^{(\mathrm{m})}=2 \mathrm{~m}\left(\sum_{I} n_{I}+1 / 2\right) \psi_{\mathbf{n}}^{(\mathrm{m})}$. Introducing a Schwinger parameter $s$ and performing the discrete sums and the $p_{+}, p_{-}$integrals one gets

$$
G_{c}\left(x_{1}, x_{2}\right)=e^{i c \mu r+} G_{0}\left(x_{1}, x_{2}\right)
$$

with

$$
G_{0}\left(x_{1}, x_{2}\right)=i\left(\frac{\mu r^{+}}{\sin \mu r^{+}}\right)^{4} \int_{0}^{\infty} \frac{d s}{(4 \pi i s)^{5}} e^{-\frac{\sigma+i \epsilon}{4 i s}}
$$

with

$$
\sigma=2 r^{+} r^{-}+\frac{\mu r^{+}}{\sin \mu r^{+}}\left(\left(x_{1}^{I} x_{1}^{I}+x_{2}^{I} x_{2}^{I}\right) \cos \mu r^{+}-2 x_{1}^{I} x_{2}^{I}\right)
$$


and we have defined $r^{\mu} \equiv x_{1}^{\mu}-x_{2}^{\mu}$. The quantity $\sigma$ is proportional to the invariant distance squared $\Phi$ :

$$
\sigma=\frac{\mu r^{+}}{\sin \mu \rho^{+}} \Phi
$$

The integral in (A.9) can be performed to give

$$
G_{0}\left(x_{1}, x_{2}\right)=\frac{3}{2 \pi^{5}(\Phi+i \epsilon)^{4}}
$$

in agreement with $[35,22]$. The limit $\mu \rightarrow 0$ yields the Feynman propagator in Minkowski space. In the calculation of D-brane interaction energies, we will need the integrated propagator $I_{c}^{9-p}\left(r^{+}, r^{-}\right)$over $p+1$ longitudinal $x^{I}$ directions with the transverse $x^{I}$ set to zero. From (A.9) one gets

$$
\begin{aligned}
I_{c}^{9-p}\left(r^{+}, r^{-}\right) & =e^{i c \mu r^{+}} I_{0}^{9-p}\left(r^{+}, r^{-}\right) \\
& =\frac{1}{4 \pi} e^{i c \mu r^{+}} \frac{\left(2 \pi r^{-}\right)^{p-3} \mu^{3-p}}{\sin ^{4} \mu r^{+}} \Gamma(3-p) .
\end{aligned}
$$

\section{A.3 Massless modes in $P P_{6} \times R^{4}$}

We use the following index conventions:

$$
\begin{array}{rl}
\mu, \nu, \ldots=0, \ldots, 9 & \mathrm{SO}(9,1) \text { vector indices } \\
I, J, \ldots=1, \ldots 8 & \mathrm{SO}(8) \text { vector indices } \\
i, j, \ldots=1,2 & \mathrm{U}(1) \text { vector indices } \\
i^{\prime}, j^{\prime}, \ldots=3,4 & \mathrm{U}^{\prime}(1) \text { vector indices } \\
a, b, \ldots=5, \ldots, 8 & \mathrm{SO}(4) \text { vector indices }
\end{array}
$$

It is convenient to work with complex coordinates $z, w$ instead of $x^{i}$ and $x^{i^{\prime}}$ :

$$
\begin{aligned}
z & =x^{1}+i x^{2} \\
w & =x^{3}+i x^{4}
\end{aligned}
$$

The nonvanishing $P P_{6} \times R^{4}$ background fields are then given by

$$
\begin{aligned}
d s^{2} & =2 d x^{+} d x^{-}-\mu^{2}(z \bar{z}+w \bar{w})\left(d x^{+}\right)^{2}+d z d \bar{z}+d w d \bar{w}+d x^{a} d x^{a} \\
R_{z++\bar{z}} & =-\frac{1}{2} \mu^{2} \quad R_{w++\bar{w}}=-\frac{1}{2} \mu^{2} \quad R_{++}=4 \mu^{2} \\
F_{+z \bar{z}} & =F_{+w \bar{w}}=i \mu
\end{aligned}
$$

In light-cone gauge, the $U(1) \times U^{\prime}(1) \times S O(4)$ subgroup of the background symmetry group is manifest with $z$ and $w$ carrying charge -1 under $U(1)$ and $U^{\prime}(1)$ respectively 


\begin{tabular}{|c|c|c|c|c|}
\hline$\psi$ & & linear combination & $\mathrm{c}$ & irrep \\
\hline$\hat{h}_{z z}$ & $=$ & $2 h_{z z}^{T}$ & 0 & $\mathbf{1}_{(2,0)}$ \\
\hline$\tilde{h}_{w w}$ & $=$ & $2 h_{w w}^{T}$ & 0 & $\mathbf{1}_{(2,0)}$ \\
\hline$h_{a b}^{\perp}$ & $=$ & $\frac{1}{\sqrt{2}}\left(h_{a b}^{T}+\delta_{a b}\left(h_{z \bar{z}}^{T}+h_{w \bar{w}}^{T}\right)\right)$ & 0 & $9_{(0,0)}$ \\
\hline$\tilde{a}_{a b}$ & $=$ & $\frac{1}{\sqrt{2}} a_{a b}$ & 0 & $6_{(0,0)}$ \\
\hline$H_{z w}^{ \pm}$ & $=$ & $2\left(h_{z w}^{T} \pm a_{z w}\right)$ & 0 & $\mathbf{1}_{(\mathbf{1}, \mathbf{1})}$ \\
\hline$H_{z \bar{w}}^{ \pm}$ & $=$ & $2\left(h_{z \bar{w}}^{T} \pm a_{z \bar{w}}\right)$ & \pm 2 & $\mathbf{1}_{(\mathbf{1},-1)}$ \\
\hline$H_{0}$ & $=$ & $\sqrt{2}\left(h_{z \bar{z}}^{T}+h_{w \bar{w}}^{T}\right)+\frac{1}{\sqrt{2}} \phi$ & 0 & $\mathbf{1}_{(0,0)}$ \\
\hline$H$ & $=$ & $2 h_{z \bar{z}}^{T}-\frac{1}{2} \phi+2 a_{z \bar{z}}$ & 2 & $\mathbf{1}_{(0,0)}$ \\
\hline$H^{\prime}$ & $=$ & $2 h_{w \bar{w}}^{T}-\frac{1}{2} \phi+2 a_{w \bar{w}}$ & 2 & $\mathbf{1}_{(0,0)}$ \\
\hline$H_{a z}^{ \pm}$ & $=$ & $\sqrt{2}\left(h_{a z}^{T} \pm a_{a z}\right)$ & $\mp 1$ & $4_{(1,0)}$ \\
\hline$H_{a w}^{ \pm}$ & $=$ & $\sqrt{2}\left(h_{a w}^{T} \pm a_{a w}\right)$ & $\mp 1$ & $4_{(0,1)}$ \\
\hline$G_{0}$ & $=$ & $\frac{1}{\sqrt{2}}\left(a-4 a_{z \bar{z} w \bar{w}}\right)$ & 0 & $\mathbf{1}_{(0,0)}$ \\
\hline$G_{0}^{\prime}$ & $=$ & $-i \sqrt{2}\left(b_{z \bar{z}}-b_{w \bar{w}}\right)$ & 0 & $\mathbf{1}_{(0,0)}$ \\
\hline$G$ & $=$ & $\sqrt{2}\left(b_{z \bar{z}}+b_{w \bar{w}}\right)+\frac{1}{\sqrt{2}}\left(a+4 a_{z \bar{z} w \bar{w}}\right)$ & 2 & $\mathbf{1}_{(0,0)}$ \\
\hline
\end{tabular}

Table 2: Decoupled massless fields in $P P_{6} \times R^{4}$.

and $x^{a}$ transforming as a vector under $S O(4)$. We can again organize the massless modes into decoupled fields $\psi$ which transform in irreps of $U(1) \times U^{\prime}(1) \times S O(4)$ and whose contribution to the action is characterized by an integer $c$ as in (A.7), where the scalar Laplacian is now $\square=2 \partial_{+} \partial_{-}+\mu^{2}(z \bar{z}+w \bar{w}) \partial_{-}^{2}+4 \partial_{z} \partial_{\bar{z}}+4 \partial_{w} \partial_{\bar{w}}+\partial_{a} \partial_{a}$. The results of this heartwarming calculation are summarized in table 2 . It displays the fields $\psi$, their definition in terms of the original fluctuations (A.3), their value of $c$ and their irrep of $U(1) \times U^{\prime}(1) \times S O(4)$. We use the notation $\mathbf{d}_{\left(\mathbf{q}, \mathbf{q}^{\prime}\right)}$ for the $d$-dimensional representation of $S O(4)$ with charges $\left(q, q^{\prime}\right)$ under $U(1) \times U^{\prime}(1)$.

\section{A.4 Massless propagators in $P P_{6} \times R^{4}$}

The Feynman propagator $G_{c}\left(x_{1}, x_{2}\right)$ corresponding to the operator $\square-2 i \mu c \partial_{-}$can be written as

$$
-i \sum_{\mathbf{n}} \int \frac{d p_{+} d p_{-} d^{4} p_{a}}{(2 \pi)^{6}} \frac{e^{i\left(p_{+}\left(x_{1}^{+}-x_{2}^{+}\right)+p_{-}\left(x_{1}^{-}-x_{2}^{-}\right)+p_{a}\left(x_{1}^{a}-x_{2}^{a}\right)\right)} \psi_{\mathbf{n}}^{\left(\mu p_{-}\right)}\left(x_{1}^{A}\right) \psi_{\mathbf{n}}^{\left(\mu p_{-}\right)}\left(x_{2}^{A}\right)}{2 p_{+} p_{-}+\mu p_{-} \sum_{A}\left(n_{A}+\frac{1}{2}\right)+2 c \mu p_{-}+p_{a} p_{a}-i \epsilon}
$$

Here, $A=1 \ldots 4, \mathbf{n}=\left(n_{1}, n_{2}, n_{3}, n_{4}\right)$ and $\psi_{\mathbf{n}}^{(\mathrm{m})}$ is a product of harmonic oscillator eigenfunctions satisfying $\left(-\partial_{i} \partial_{i}-\partial_{i^{\prime}} \partial_{i^{\prime}}+\mathrm{m}^{2}\right) \psi_{\mathbf{n}}^{(\mathrm{m})}=2 \mathrm{~m}\left(\sum_{A} n_{A}+1 / 2\right) \psi_{\mathbf{n}}^{(\mathrm{m})}$. 
Introducing a Schwinger parameter $s$ and performing the discrete sums and the $p_{+}, p_{-}, p_{a}$ integrals one gets

$$
G_{c}\left(x_{1}, x_{2}\right)=e^{i c \mu r^{+}} G_{0}\left(x_{1}, x_{2}\right)
$$

with

$$
G_{0}\left(x_{1}, x_{2}\right)=i\left(\frac{\mu r^{+}}{\sin \mu r^{+}}\right)^{2} \int_{0}^{\infty} \frac{d s}{(4 \pi i s)^{5}} e^{-\frac{\sigma+i \epsilon}{4 i s}}
$$

with

$$
\begin{aligned}
\sigma & =2 r^{+} r^{-}+r^{a} r^{a} \\
& +\frac{\mu r^{+}}{\sin \mu r^{+}}\left(\left(x_{1}^{i} x_{1}^{i}+x_{1}^{i^{\prime}} x_{1}^{i^{\prime}}+x_{2}^{i} x_{2}^{i}+x_{2}^{i^{\prime}} x_{2}^{i^{\prime}}\right) \cos \mu r^{+}-2\left(x_{1}^{i} x_{2}^{i}+x_{1}^{i^{\prime}} x_{2}^{i^{\prime}}\right)\right)
\end{aligned}
$$

and we have defined $r^{\mu} \equiv x_{1}^{\mu}-x_{2}^{\mu}$. In the calculation of D-brane interaction energies, we need the integrated propagator, denoted by $I_{c}^{9-p}$, over $p+1$ longitudinal D-brane directions (which we take be a subset of the pp-wave directions $x^{1}, \ldots x^{4}$ ) and with the remaining pp-wave coordinates set to zero:

$$
\begin{aligned}
I_{c}^{9-p}\left(r^{+}, r^{-}, r^{a}\right) & =e^{i c \mu r^{+}} I_{0}^{9-p}\left(r^{+}, r^{-}, r^{a}\right) \\
& =\frac{1}{4 \pi} e^{i c \mu r^{+}} \frac{\left(\pi r^{2}\right)^{p-3}\left(\mu r^{+}\right)^{1-p}}{\sin ^{2} \mu r^{+}} \Gamma(3-p)
\end{aligned}
$$

where $r^{2} \equiv 2 r^{+} r^{-}+r^{a} r^{a}$.

\section{References}

[1] J. Polchinski, "Dirichlet-Branes and Ramond-Ramond Charges," Phys. Rev. Lett. 75, 4724 (1995) [arXiv:hep-th/9510017].

[2] R. I. Nepomechie, "Magnetic Monopoles From Antisymmetric Tensor Gauge Fields," Phys. Rev. D 31, 1921 (1985).

C. Teitelboim, "Monopoles Of Higher Rank," Phys. Lett. B 167, 69 (1986).

[3] K. Dasgupta, D. P. Jatkar and S. Mukhi, "Gravitational couplings and Z(2) orientifolds," Nucl. Phys. B 523, 465 (1998) [arXiv:hep-th/9707224].

[4] C. P. Bachas, P. Bain and M. B. Green, "Curvature terms in D-brane actions and their M-theory origin," JHEP 9905, 011 (1999) [arXiv:hepth/9903210]. 
[5] J. A. Harvey, S. Kachru, G. W. Moore and E. Silverstein, "Tension is dimension," JHEP 0003, 001 (2000) [arXiv:hep-th/9909072].

[6] C. Bachas, M. R. Douglas and C. Schweigert, "Flux stabilization of Dbranes," JHEP 0005, 048 (2000) [arXiv:hep-th/0003037].

[7] M. Blau, J. Figueroa-O'Farrill, C. Hull and G. Papadopoulos, "A new maximally supersymmetric background of IIB superstring theory," JHEP 0201, 047 (2002) [arXiv:hep-th/0110242].

M. Blau, J. Figueroa-O'Farrill, C. Hull and G. Papadopoulos, "Penrose limits and maximal supersymmetry," arXiv:hep-th/0201081.

[8] D. Berenstein, J. Maldacena and H. Nastase, "Strings in flat space and pp waves from $\mathcal{N}=4$ super Yang Mills," arXiv:hep-th/0202021.

[9] R. Penrose, "Any space-time has a plane wave as a limit," in Differential geometry and relativity, pp. 271-275, Reidel, Dordrecht, 1976.

[10] R. Güven, "Plane wave limits and T-duality," Phys. Lett. B482 (2000) 255, [arXiv: hep-th/0005061].

[11] K. Skenderis and M. Taylor, "Branes in AdS and pp-wave spacetimes," JHEP 0206, 025 (2002) [arXiv:hep-th/0204054].

[12] R. R. Metsaev, "Type IIB Green-Schwarz superstring in plane wave Ramond-Ramond background," arXiv:hep-th/0112044.

[13] R. R. Metsaev and A. A. Tseytlin, "Exactly solvable model of superstring in plane wave Ramond-Ramond background," arXiv:hep-th/0202109.

[14] A. Dabholkar and S. Parvizi, "Dp branes in pp-wave background," Nucl. Phys. B 641, 223 (2002) [arXiv:hep-th/0203231].

[15] M. Billo' and I. Pesando, "Boundary states for GS superstrings in an Hpp wave background," arXiv:hep-th/0203028.

[16] Y. Michishita, "D-branes in NSNS and RR pp-wave backgrounds and Sduality," JHEP 0210, 048 (2002) [arXiv:hep-th/0206131].

[17] K. Skenderis and M. Taylor, "Open strings in the plane wave background. I: Quantization and symmetries," arXiv:hep-th/0211011.

K. Skenderis and M. Taylor, "Open strings in the plane wave background. II: Superalgebras and spectra," JHEP 0307, 006 (2003) [arXiv:hepth/0212184]. 
D. Z. Freedman, K. Skenderis and M. Taylor, "Worldvolume supersymmetries for branes in plane waves," arXiv:hep-th/0306046.

[18] Y. Hikida and S. Yamaguchi, "D-branes in pp-waves and massive theories on worldsheet with boundary," JHEP 0301, 072 (2003) [arXiv:hepth/0210262].

[19] M. R. Gaberdiel, M. B. Green, S. Schafer-Nameki and A. Sinha, "Oblique and curved D-branes in IIB plane-wave string theory," arXiv:hepth/0306056.

G. Sarkissian and M. Zamaklar, "Diagonal D-branes in product spaces and their Penrose limits," arXiv:hep-th/0308174.

[20] A. Kumar, R. R. Nayak and Sanjay, "D-brane solutions in pp-wave background," Phys. Lett. B 541, 183 (2002) [arXiv:hep-th/0204025].

D. s. Bak, "Supersymmetric branes in PP wave background," Phys. Rev. D 67, 045017 (2003) [arXiv:hep-th/0204033].

P. Bain, P. Meessen and M. Zamaklar, "Supergravity solutions for D-branes in Hpp-wave backgrounds," Class. Quant. Grav. 20, 913 (2003) [arXiv:hep-th/0205106].

M. Alishahiha and A. Kumar, "D-brane solutions from new isometries of pp-waves," Phys. Lett. B 542, 130 (2002) [arXiv:hep-th/0205134].

S. Seki, "D5-brane in Anti-de Sitter space and Penrose limit," Phys. Lett. B 542, 162 (2002) [Phys. Lett. B 542, 165 (2002)] [arXiv:hep-th/0205266].

L. F. Alday and M. Cirafici, "An example of localized D-branes solution on pp-wave backgrounds," JHEP 0305, 006 (2003) [arXiv:hep-th/0301253].

J. w. Kim, B. H. Lee and H. S. Yang, "Superstrings and D-branes in plane wave," Phys. Rev. D 68, 026004 (2003) [arXiv:hep-th/0302060].

[21] O. Bergman, M. R. Gaberdiel and M. B. Green, "D-brane interactions in type IIB plane-wave background," JHEP 0303, 002 (2003) [arXiv:hepth/0205183].

[22] M. R. Gaberdiel and M. B. Green, "The D-instanton and other supersymmetric D-branes in IIB plane-wave string theory," arXiv:hep-th/0211122.

[23] C. V. Johnson and H. G. Svendsen, "D-brane anti-D-brane forces in plane wave backgrounds: A fall from grace," JHEP 0305, 055 (2003) [arXiv:hepth/0303255].

[24] A. Sinha and N. V. Suryanarayana, "Tadpole analysis of orientifolded plane-waves," JHEP 0211, 026 (2002) [arXiv:hep-th/0209247]. 
[25] A. B. Hammou, JHEP 0211, 028 (2002) [arXiv:hep-th/0209265].

[26] A. Karch and L. Randall, "Open and closed string interpretation of SUSY CFT's on branes with boundaries," JHEP 0106, 063 (2001) [arXiv:hepth/0105132].

[27] O. DeWolfe, D. Z. Freedman and H. Ooguri, "Holography and defect conformal field theories," Phys. Rev. D 66, 025009 (2002) [arXiv:hepth/0111135].

[28] C. Bachas, J. de Boer, R. Dijkgraaf and H. Ooguri, "Permeable conformal walls and holography," JHEP 0206, 027 (2002) [arXiv:hep-th/0111210].

[29] J. Erdmenger, Z. Guralnik and I. Kirsch, "Four-dimensional superconformal theories with interacting boundaries or defects," Phys. Rev. D 66, 025020 (2002) [arXiv:hep-th/0203020].

[30] N. R. Constable, J. Erdmenger, Z. Guralnik and I. Kirsch, "Intersecting D3-branes and holography," arXiv:hep-th/0211222.

[31] D. Berenstein, E. Gava, J. M. Maldacena, K. S. Narain and H. Nastase, "Open strings on plane waves and their Yang-Mills duals," arXiv:hepth/0203249.

[32] P. Lee and J. w. Park, "Open strings in PP-wave background from defect conformal field theory," Phys. Rev. D 67, 026002 (2003) [arXiv:hepth/0203257].

[33] B. S. DeWitt, "Dynamical Theory Of Groups And Fields," in DeWitt C. (ed.) et. al.: Relativity Groups and Topology, 725 .

[34] N.D.Birrell and P.C.W. Davies, "Quantum Fields In Curved Space", Cambridge University Press 1982.

[35] S. D. Mathur, A. Saxena and Y. K. Srivastava, "Scalar propagator in the pp-wave geometry obtained from $\operatorname{AdS}(5)$ x S(5)," Nucl. Phys. B 640, 367 (2002) [arXiv:hep-th/0205136].

[36] W. Siegel, "Fields," arXiv:hep-th/9912205. 